\title{
Ultrasound Triggered Drug Delivery with Liposomal Nested Microbubbles
}

N. Wallace ${ }^{1}$, S. P. Wrenn* ${ }^{1}$

\section{Abstract}

When ultrasound contrast agent microbubbles are nested within a liposome, damage to the liposome membrane caused by both stable and inertial cavitation of the microbubble allows for release of the aqueous core of the liposome. Triggered release was not accomplished unless microbubbles were present within the liposome. Leakage was tested using fluorescence assays developed specifically for this drug delivery vehicle and qualitative measurements using an optical microscope. These studies were done using a $1 \mathrm{MHz}$ focused ultrasound transducer while varying parameters including peak negative ultrasound pressure, average liposome diameter, and microbubble concentration. Two regimes exist for membrane disruption caused by cavitating microbubbles. A faster release rate, as well as permanent membrane damage are seen for samples exposed to high pressure (2.06 to $3.74 \mathrm{MPa}$ ). A slower release rate and dilation/ temporary poration are characteristic of stable cavitation for low pressure studies (0.54 to $1.74 \mathrm{MPa})$.

Keywords: Ultrasound, microbubble, drug delivery, cavitation, sonoporation

\section{Introduction}

The first description of swollen phospholipid systems was introduced by Bangham et al. in 1965 [1]. Over the next few years, early pioneers such as Gregoriadis et al. established the use of liposomes as a drug entrapment vehicle to be used for drug delivery systems [2]. Liposomes offer the benefit of a biodegradable drug delivery vehicle suitable for both hydrophilic and lipophilic drugs. They have been shown to retain their contents and remain stable during circulation within the body [3]. However, because exposure of all cells in the body to a systematically administered chemotherapy drug is the main cause of harmful side effects, a triggered release system is desirable. For optimal therapeutic activity, the drug must first be delivered to the diseased site and then be released at a sufficient rate. This requires the drugdelivery vehicle to change its state from a relatively stable structure with a very slow or nonexistent leak rate to an unstable structure which allows for release of its contents. An external trigger eliminates many of the negative side effects associated with pure biochemical targeting. Ultrasound is an attractive candidate in controlled release applications because it is non-ionizing and non-invasive yet still able to penetrate body tissue [4].

Gas filled particles known as microbubbles are small enough to pass through circulation and ideal for ultrasonic release vehicles. Microbubbles increase the absorption of sonic energy through a mechanism known as cavitation [7]. An ultrasonic wave causes the microbubbles to oscillate with a wall velocity on the order of tens to hundreds of meters per second [8]. At relatively low pressures above an approximate threshold of $0.3-0.4 \mathrm{MPa}$ at $2.5 \mathrm{MHz}$, these sustained oscillations are referred to as stable cavitation [9]. As the peak negative pressure (PNP) increases, the microbubble will expand so large, that upon subsequent contraction it implodes, generally occurring when the radius reaches twice its resting radius [10]. The destruction of the microbubble occurs at its inertial cavitation threshold, which is the peak negative pressure at which the microbubble implodes and fractures, around $1.5 \mathrm{MPa}$ at $2.5 \mathrm{MHz}$ for microbubbles

${ }^{1}$ Department of Chemical Engineering, Drexel University, Philadelphia, PA, USA

*Corresponding author, email spw22@drexel.edu, phone +1 215-895-6694 
with radii from 0.1 to $10 \mu \mathrm{m}[9,11]$. The inertial cavitation varies based on acoustic power, frequency, and microbubble shell dynamics.

Combination of ultrasound and contrast agent microbubbles is believed to alter liposome membrane permeability [12-16] causing transient formation of pores in the membrane, known as sonoporation $[17,18]$. Previous attempts at formulating these vehicles have very low yields, lack precision with gas and drug encapsulation, stability and internal geometry [19]. To overcome these obstacles, we are presenting a means for ultrasound triggered drug delivery by nesting a solution of microbubbles within the aqueous core of a liposome which protects both gas microbubbles and aqueous contents. When exposed to ultrasound, the microbubble oscillates which induces microstreaming of the surrounding fluid and disrupts the membrane. In this case, poration of the membrane facilitates release of the drug from the liposome to a nearby structure, which we refer to as reverse sonoporation. In reverse sonoporation, the direction of transport and perhaps also the mechanism of poration is different than traditional sonoporation [21]. Using focused ultrasound, only the desired region within the focal zone of the transducer will receive enough energy to release the drug, reducing systemic side effects.

This study evaluates a potential drug delivery vehicle which involves microbubble cavitationinduced leakage of a fluorescent dye nested in the aqueous core of a liposome. $\mathrm{SF}_{6}$ microbubbles coated by a 95\% DSPC/ 5\% DSPE PEG-3000 monolayer plus calcein, a self quenching fluorophore, are nested within a liposome (70\% Egg-PC/ 20\% cholesterol/ 10\% triolein) using a double emulsion technique. Calcein leakage is monitored as a function of ultrasound pressure (0.54 MPa to 3.74 MPa), ultrasound exposure (0-2200 seconds), average liposome shell diameter $(4.74$ to $6.04 \mu \mathrm{m})$, and concentration of microbubbles used during formulation $\left(2 \times 10^{8}\right.$ to $8 \times 10^{8}$ $\mathrm{MB} / \mathrm{mL}$ ). In vitro experiments at high frequency are carried out with a $7.5 \mathrm{~cm}$ focused, $1 \mathrm{MHz}$ transducer submerged in a glass beaker containing the sample insonated with a high voltage pulser generating a signal consisting of 4 cycle pulses with a pulse repetition frequency of $5 \mathrm{~Hz}$. Fluorescent measurements are taken at 200 second intervals ranging from 0 up to 2200 seconds.

\section{Experimental}

\subsection{Materials}

Polyvinyl alcohol (PVA) supplied by Sigma-Aldrich (St. Louis, MO, USA) is reported to have a MW of 85,000-124,000 and is 99+\% hydrolyzed. The lipids, 1,2-distearoyl-sn-glycero-3phosphocholine (DSPC), 1,2-distearoyl-sn-glycero-3-phosphoethanolamine-N[methoxy(polyethyleneglycol)-3000] (DSPE-PEG3000), 1,2,3-oleoyl-glycerol (Triolein), and L$\alpha$-phosphatidylcholine (Egg-PC) were purchased from Avanti Polar Lipids (Alabaster, AL, USA,). Sulfur hexafluoride $\left(\mathrm{SF}_{6}\right)$ and nitrogen gas $\left(\mathrm{N}_{2}\right)$ were purchased from Airgas (Allentown PA, USA). Cholesterol, calcein, cobalt (II) chloride $\left(\mathrm{CoCl}_{2}\right)$ and Triton $\mathrm{X}-100$ were purchased from Sigma Aldrich (St. Louis, MO) and chloroform was purchased from Fisher Scientific (Fair Lawn, NJ). All products were used without further purification. All other reagents used were of analytical grade.

\subsection{Microbubble formation}


Microbubbles were prepared as described previously [22]. Lipid film solutions of 95 mole \% DSPC in chloroform and 5 mole \% DSPE-PEG3000 in chloroform both with a concentration of $25 \mathrm{mg} / \mathrm{mL}$, were mixed together in a $20 \mathrm{~mL}$ scintillation vial and spin dried under a stream of nitrogen for 15 minutes or until the chloroform had evaporated. The combination of spinning while drying permits a uniform film on the bottom of the vial. After drying, the film solution was placed in a vacuum oven for 24 hours at $40^{\circ} \mathrm{C}$ to ensure that residual chloroform was removed from the film. After the drying stages were completed, the film was rehydrated with aqueous phosphate buffered saline (PBS) and sonicated using a $20 \mathrm{kHz}$ Misonix XL2020 probe tip sonicator (Misonix Inc., Farmingdale, NY) operating at $20 \%$ amplitude for 3 minutes. Sonication at this frequency elevates the temperature of lipid above the gel phase transition temperature of $55^{\circ} \mathrm{C}$ and also ensures that the lipid mixture in solution is fully dissolved. $1.5 \mathrm{~mL}$ aliquots of solution were placed in $2 \mathrm{~mL}$ serum vials and sealed with a rubber stopper and crimp cap. The air in the headspace of the vial was replaced with $\mathrm{SF}_{6}$ by method of vacuum exchange and stored at $2^{\circ} \mathrm{C}$. Before experimentation, a Vialmix shaker (Lantheus Medical Imaging, North Billerica, MA, USA) was used for 45 seconds to disperse the gas phase within the solution. This technique successfully produces stable microbubbles at a concentration of approximately $1 \times 10^{9}$ microbubbles/mL with an average diameter of $1.5 \mu \mathrm{m}$.

\subsection{Nested Liposome Synthesis}

A double emulsion technique is used to encapsulate microbubbles plus calcein, a self quenching fluorophore, within the liposome shell. Nested liposomes were prepared with an Egg-PC/ cholesterol/ triolein lipid mixture to form the unilamellar vesicle using the water/oil/water $\left(\mathrm{W}_{1} / \mathrm{O} / \mathrm{W}_{2}\right)$ emulsion technique $[23,24]$. A solution of $0.5 \mathrm{~mL}$ of the previously described microbubbles and $0.5 \mathrm{~mL}$ of $0.1 \mathrm{mM}$ calcein solution (internal aqueous phase $\mathrm{W}_{1}$ ) was added to $1 \mathrm{~mL}$ of a lipid solution containing $733.5 \mu \mathrm{L}$ of chloroform, $185 \mu \mathrm{L}$ of $25 \mathrm{mg} / \mathrm{mL}$ Egg-PC in chloroform, $50.5 \mu \mathrm{L}$ of $50 \mathrm{mg} / \mathrm{mL}$ cholesterol in chloroform, and $31.0 \mu \mathrm{L}$ of $100 \mathrm{mg} / \mathrm{mL}$ triolein in chloroform (intermediate organic phase $\mathrm{O}$ ). The solution of microbubbles/calcein and lipid mixture was homogenized with the Polytron PT3100 (Kinematica Inc, Lucerne, Switzerland) for 1 minute. The speed of the homogenizer was varied between $5000 \mathrm{rpm}$ and $15000 \mathrm{rpm}$ to control the size of the unilamellar vesicle. After 1 minute, the solution was added to $8 \mathrm{~mL}$ of $2 \%$ PVA in water (outer aqueous phase $\mathrm{W}_{2}$ ) and further homogenized for 2 minutes at the same speed as in the previous step. After homogenization of solution, the contents were added to $8 \mathrm{~mL}$ of the $\mathrm{W}_{2}$ phase and placed on a magnetic stir plate at $300 \mathrm{rpm}$ for 24 hours so that the chloroform evaporates from the solution, leaving aqueous phase $\mathrm{W}_{1}$ nested within the lipid bilayer. External calcein was quenched with $50 \mu \mathrm{L}$ of $40 \mathrm{mM} \mathrm{CoCl}_{2}$. Figure 1a shows an image of the liposome shell with microbubbles nested inside, while Figure $1 \mathrm{~b}$ is a fluorescent image of the same sample to show that only the external calcein has been quenched while the calcein entrapped within the liposome accounts for the fluorescence intensity of the sample. 
a)

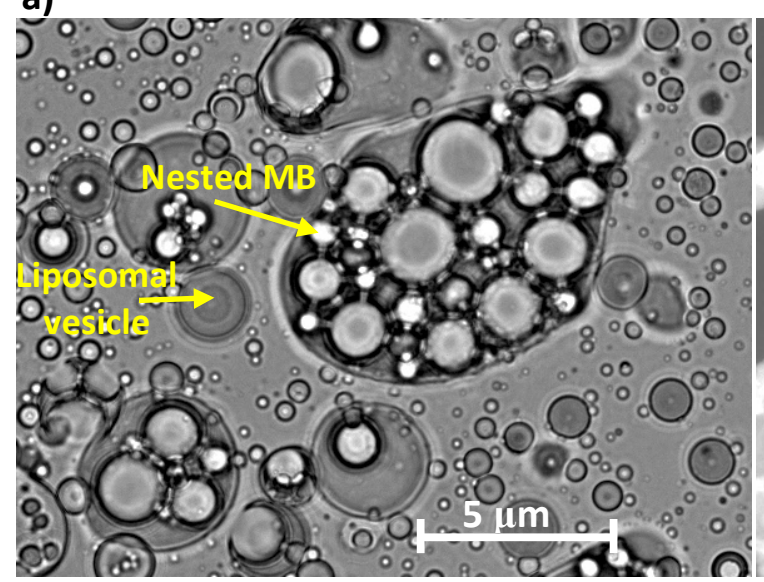

b)

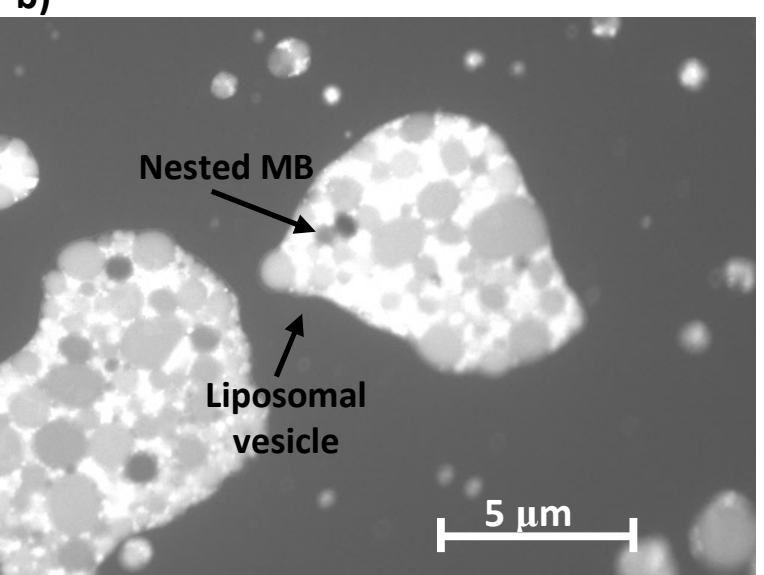

Figure 1: Microscope images taken at 100X magnification shown for a) DSPC microbubbles containing 5 mol \% DSPE-PEG3000 nested within a liposomal vesicle, b) image taken with a fluorescent microscope (Axioskop 2; Carl Zeiss, Heidenheim, Germany) of the same formulation after external calcein has been quenched.

\subsubsection{Variation of Homogenizer Speed during Formulation}

The average diameter of the liposome was varied systematically by adjusting the homogenizing speed. Homogenizing speed is a parameter of primary importance in the emulsification step because it provides the energy to disperse the oil phase in aqueous phase. To determine a size range for a population of nested microbubbles at each homogenizer speed, samples of the selected formulation are prepared by the method listed above and then diluted for a more accurate reading. The size distribution was obtained from a Coulter Multisizer II (BeckmannCoulter, Brea, CA) for a $50 \mu \mathrm{L}$ sample of nested microbubbles diluted by a factor of 100 . These results are shown below in Table I.

Table 1: Size distribution of each nested microbubble population as determined from the Coulter Multisizer II (Beckmann-Coulter, Brea, CA) for a $50 \mu \mathrm{L}$ sample.

\begin{tabular}{|c|c|c|}
$\begin{array}{c}\text { Homogenizer Speed } \\
\text { (RPM) }\end{array}$ & $\begin{array}{c}\text { Liposome Diameter }(\boldsymbol{\mu m}) \\
\text { (Mean } \pm \text { SD) }\end{array}$ & $\begin{array}{c}\text { Concentration of } \\
\text { nested MB } \\
\text { (Particles/mL) }\end{array}$ \\
\hline 5000 & $6.04 \pm 1.38$ & $5.42 \times 10^{7}$ \\
\hline 7500 & $5.78 \pm 1.07$ & $7.87 \times 10^{7}$ \\
\hline 10000 & $5.26 \pm 1.01$ & $9.04 \times 10^{7}$ \\
\hline 12500 & $5.01 \pm 0.83$ & $9.32 \times 10^{7}$ \\
\hline 15000 & $4.74 \pm 0.76$ & $9.60 \times 10^{7}$ \\
\hline
\end{tabular}

From Table 1, we conclude that shell diameter of liposome is inversely proportional to homogenizing speed; consequently an increase in homogenizing speed decreases the size of liposomes. The average liposome diameters for each homogenizing speed are the same order of magnitude owing to fact that the speed does not have as large of an effect on the flexible membrane as it would with a stiffer polymer shell used for imaging purposes (see [25]). A Beckmann-Coulter Z1 Particle Counter (Beckmann-Coulter, Brea, CA) was used to confirm that when using the same amount of material during synthesis, the number of nested microbubbles increases with homogenizer speed. 


\subsection{Ultrasound Therapy}

\subsubsection{Low frequency treatment}

Low frequency ultrasound treatment was performed using a Misonix XL2020 probe tip sonicator (Misonix Inc., Farmingdale, NY) operating at $20 \mathrm{kHz}$ using a 418 tip. The sonicator was calibrated using the Misonix tuning protocol and set to $10 \%$ output power (electrical) at a setting of 3. Peak-to-peak pressure amplitudes and spatial-peak, temporal-peak intensity $\left(\mathrm{I}_{\text {sptp }}\right)$ were measured using a Reson TC4038 hydrophone (Reson Inc., US, Goleta, CA) with a sensitivity of $-228.2 \mathrm{~dB}$ re $1 \mathrm{~V} / \mu \mathrm{Pa}$ at $20 \mathrm{kHz}$. To avoid possible damage of the probe caused by inertial cavitation, the pressure amplitude was measured at several distances between 10 and $50 \mathrm{~mm}$. The measurement data was plotted against distance and extrapolated to estimate the pressure amplitude at $1 \mathrm{~cm}$, which is the distance at which the release assays were performed. The amplitude was calculated to be approximately $1027 \pm 134 \mathrm{kPa}$, which corresponds to a spatialpeak, temporal-peak intensity of approximately $35.5 \mathrm{~W} / \mathrm{cm}^{2}$.

The liposome suspension was exposed to low frequency $(20 \mathrm{kHz})$, continuous wave ultrasound for the total exposure time of 45 minutes. Samples were placed in an ice water bath to maintain their temperature at $21 \pm 3^{\circ} \mathrm{C}$ to avoid temperature-induced permeability.

\subsubsection{High frequency treatment}

Ultrasound was delivered through an apparatus previously described [26-28]. Briefly, a $1 \mathrm{MHz}$ $7.5 \mathrm{~cm}$ focused ultrasound transducer (Olympus NDT, Waltham, MA, USA) is driven by a home built high voltage pulser. The transducer generates a peak negative pressure (PNP) ranging from 0.54 to $3.74 \mathrm{MPa}$, which was measured using a calibrated PVDF membrane hydrophone. The pulser signal consists of 1000 damped sinusoidal waveforms with a $5 \mathrm{~Hz}$ prf which is repeated for the desired total number of waveforms. These variables were controlled by a Matlab script. The face of the transducer is submerged within the $50 \mathrm{~mL}$ graduated cylinder at a height of 10 $\mathrm{cm}$ from the bottom, which guarantees that the focal point is within the sample region. The graduated cylinder contains the sample of nested microbubbles diluted with $40 \mathrm{~mL}$ of DI water. A $3 \mathrm{~cm}$ butyl rubber sheet was placed at the bottom of the glass cylinder to eliminate the presence of a standing wave. The sample chamber was placed on a stir plate at $300 \mathrm{rpm}$ so that particles receive uniform ultrasound exposure. The set up is shown in Figure 2. 


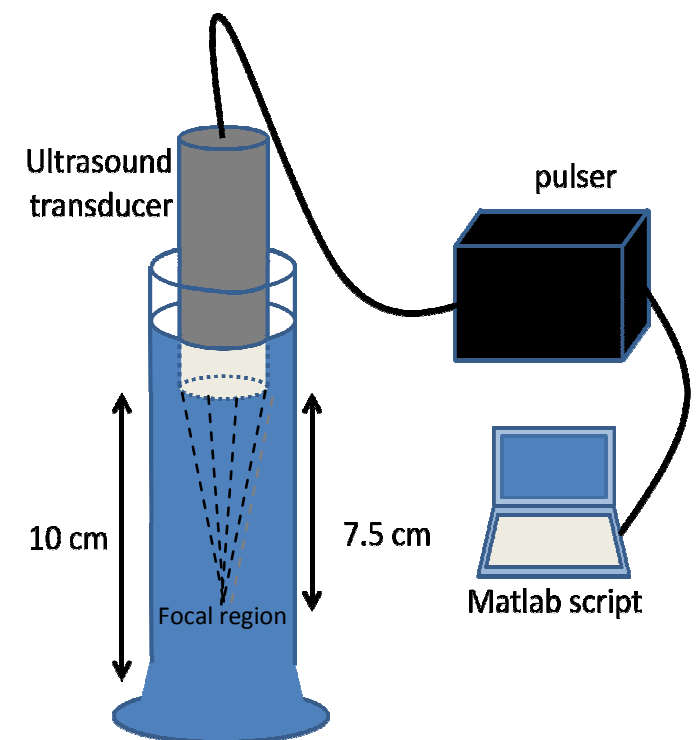

Figure 2: Diagram of high frequency ultrasound experimental set up. The $1 \mathrm{MHz}$ focused transducer is placed at the top of the graduated cylinder where the face of the transducer is $10 \mathrm{~cm}$ from the rubber sheet at the bottom of the cylinder. This permits the focal region at a distance of $7.5 \mathrm{~cm}$ from the center of the transducer to be located within the sample chamber. The transducer is driven by a voltage pulser controlled by a Matlab script.

\subsection{Fluorescence Leakage Detection}

Calcein release was detected using a cobalt quenching assay. At the beginning of each experiment $50 \mu \mathrm{L}$ of $40 \mathrm{mM} \mathrm{CoCl}_{2}$ was added to the nested microbubble solution in order to quench unencapsulated calcein. Before both high and low frequency studies, solutions were placed in the sample chamber and fluorescent measurements were taken over a $30 \mathrm{~min}$ period to confirm that there was no cause for liposome leakage due to the experimental setup, and therefore any fluorescent leakage detected was a result of ultrasound exposure. Samples were exposed to either low frequency ultrasound $(20 \mathrm{kHz})$ continuously for 45 minutes or high frequency ultrasound $(1 \mathrm{MHz})$ for a total exposure time of 40 minutes. Negative controls were performed with either no ultrasound exposure (measuring fluorescence of the solution exposed to atmospheric conditions over time) or "empty" liposomes that were made with the same contents except the microbubble solution was replaced with PBS. A $500 \mu \mathrm{L}$ aliquot of the sample was removed at each time point during the experiment and diluted with $2.5 \mathrm{~mL}$ of PBS at which point the fluorescence intensity of each sample was measured. To verify that the entire contents of the liposome was not released at steady state, the sample was treated with $1 \%$ Triton X-100 detergent to release all encapsulated calcein (positive control) and the fluorescence intensity was measured again.

A spectrophotometric technique was used to quantify leakage. Calcein emission of an initial sample before ultrasound exposure and samples from each time point were analyzed using a 4mm-path length cuvette in a Photon Technology International, Inc. (Birmingham, NJ) A-710 steady-state fluorescence spectrometer with 2-nm slit widths, 0.5 -s integration time, and 1-nm step size. The emission spectra were obtained using $\lambda_{\mathrm{ex}}=475 \mathrm{~nm}$ and $1-\mathrm{nm}$ intervals between 490 and $540 \mathrm{~nm}$. The amount of calcein remaining within the liposome core is calculated by $\frac{F_{s o n}-F_{0}}{F_{f}-F_{0}}$ where $\mathrm{F}_{\text {son }}$ is the fluorescence of the insonated sample, $\mathrm{F}_{0}$ is the initial fluorescence before 
any ultrasound exposure, and $F_{f}$ is the fluorescent measurement after complete rupture of the liposome with Triton X-100.

\subsection{Statistical analysis}

Data is presented as mean \pm standard deviation. Statistical analysis was performed with a oneway ANOVA with significance taken to be $P<0.05$ using Minitab 17 Statistical Software to detect statistically significant differences between means. A Tukey's multiple comparison test was used to determine which of the means are statistically different. Fluorescence measurements before and after ultrasound exposure were compared with negative controls.

\section{Results}

A low frequency study using a $20 \mathrm{kHz}$ sonicator was used to induce leakage of the liposomes with and without microbubbles. At an ultrasound intensity of $2.2 \mathrm{~W} \mathrm{~cm}^{-2}$ it was found that complete rupture of the liposome occurred within three minutes. Low-frequency ultrasound is known to induce inertial cavitation at relatively low intensities, even in the absence of microbubbles [29], and Figure 3 confirms this as identical leakage behavior was found for samples with and without microbubbles.

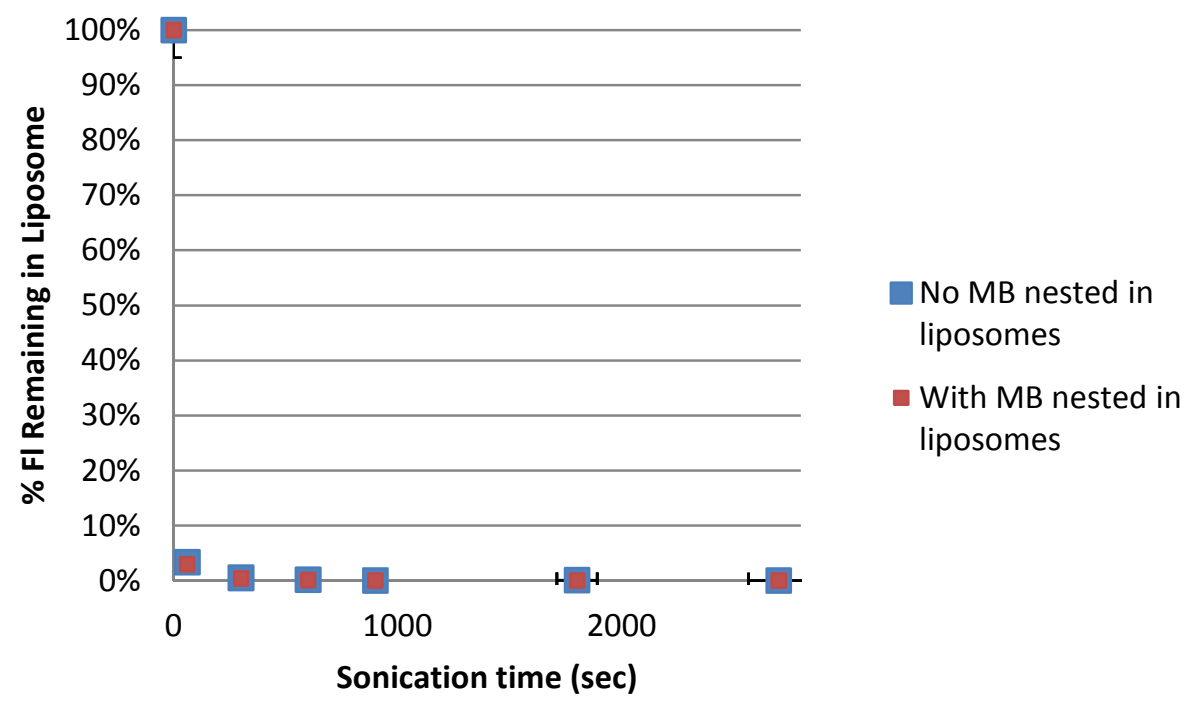

Figure 3: Fluorescence measurements for $20 \mathrm{kHz}$ continuous ultrasound exposure over the duration of 45 minutes. For this experiment, $\mathrm{PNP}=416.29 \mathrm{kPa}$ and the $\mathrm{MI}$ is 2.94. Leakage with and without microbubbles followed the same profile. The percent of fluorescence remaining was calculated according to the method presented in Section 2.5. The sample was kept in an ice bath to ensure that the temperature remained constant. This avoids the possibility that liposome poration is caused by a thermal trigger [30].

The fact that low frequency (high intensity) ultrasound can rupture liposomes without microbubbles points to generation and activation of gas bodies in the medium, which can serve as cavitation nuclei [31]. While low frequency ultrasound is useful in some clinical applications, high frequency ultrasound is more desirable for a controlled delivery application. That is, one would prefer to avoid nucleation and cavitation in the bodily fluids. As cavitation seems 
necessary for release, the question then becomes whether one can reproduce the low frequency results of Figure 3 at high frequency.

The answer is yes.

What makes this possible is the presence of microbubbles, which become essential for release at high frequency (1 MHz in this study). It was confirmed that the liposomal nested microbubbles were stable in the experimental set up and did not release any fluorescence until ultrasound was applied. As shown in Figure 4, no leakage was observed for liposomes without microbubbles. The results of Figure 4 are for liposomes with an average diameter of $5.01 \pm 0.83 \mu \mathrm{m}$ unless otherwise stated. The percent of calcein remaining within the liposomes for amplitudes 0.54 to 3.74 MPa fall between $36.57 \%$ and $62.41 \%$ respectively at steady state.

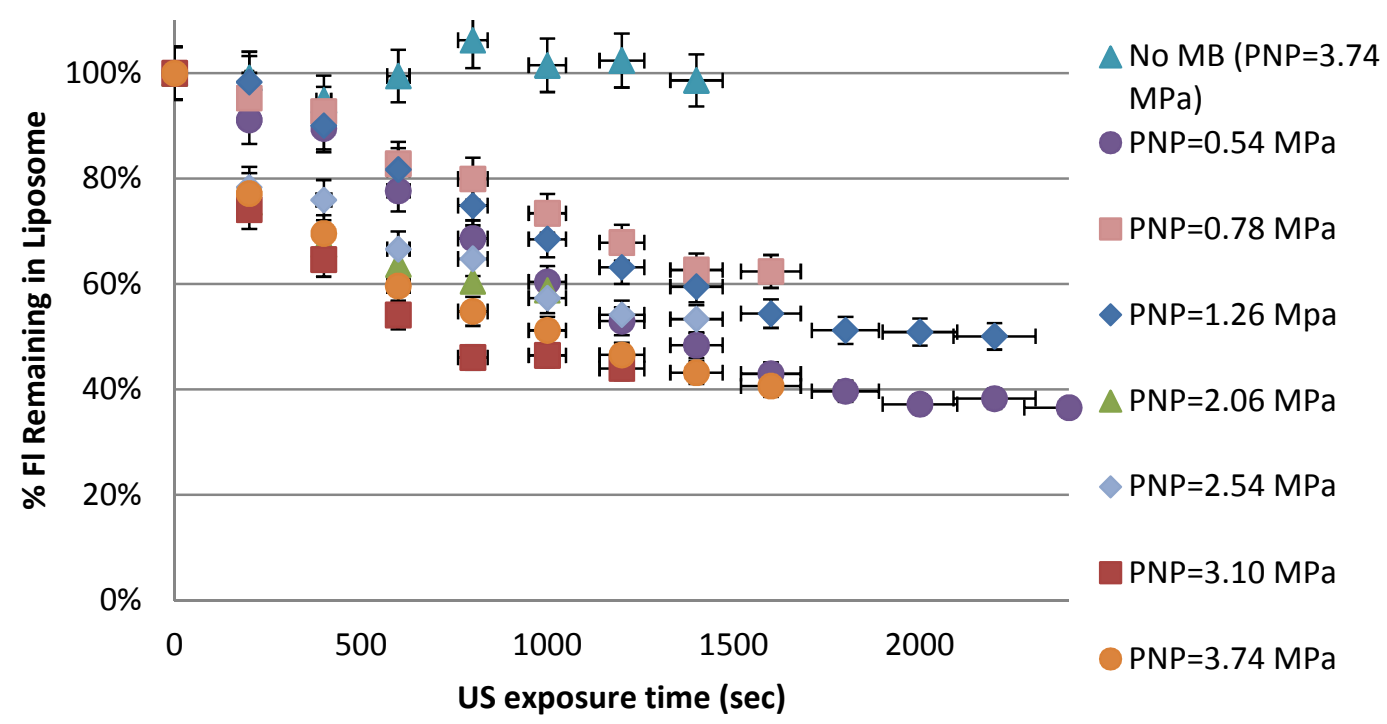

Figure 4: Graph showing the results of fluorescence measurements for $1 \mathrm{MHz}$ ultrasound transducer over an exposure time of $\mathbf{4 5} \mathrm{min}$. Note that the axis is "US exposure time" which is true for all samples except the "No US" sample. For this sample, no ultrasound was applied and the fluorescence is measured every 200 seconds, but only exposed to atmospheric conditions.

The results confirm that unless both microbubbles and ultrasound are present, no leakage occurs; thus leakage results from microbubble acoustic activity. Variation in the fluorescence value for the samples without microbubbles and without ultrasound is due to non homogeneity in the size distribution and concentration of liposomes that existed in the aliquot of sample used for fluorescence detection, as well as fluctuations caused by instrumental error. Applied pressure ranges from $0.54 \mathrm{MPa}$ to $3.74 \mathrm{MPa}$ and release from the liposome core is accomplished at each pressure when microbubbles are present. The leakage behavior at each pressure is similar in profile, but initial leakage rate is dependent on peak negative pressure.

For clarification of the interactions between microbubble oscillations and membrane disruption, it is necessary to differentiate between dilation/ temporary induced poration, where leakage of the liposome content occurs only while ultrasound is being applied, and persisting pore 
formation, where we would continue to see leakage of the fluorescent dye after ultrasound is removed. For this study, ultrasound was repeatedly applied for $400 \mathrm{sec}$ at both low ( $\mathrm{PNP}=0.54$ $\mathrm{MPa})$ and high $(\mathrm{PNP}=3.74 \mathrm{MPa})$ pressure and then removed for $120 \mathrm{sec}$. During the $120 \mathrm{sec}$ rest interval the ultrasound transducer is removed and the liposomes are allowed to stir in the sample chamber without ultrasound exposure. The application and subsequent removal was repeated four times for a total of $1600 \mathrm{sec}$ of ultrasound exposure. Fluorescence was measured before and after each interval of ultrasound application. Figure 5 shows that at the lower applied pressure of $\mathrm{PNP}=0.54 \mathrm{MPa}$, leakage ceases with removal of ultrasound and the fluorescence value does not change during the 120 seconds between intervals of ultrasound application. At the higher pressure amplitude of $\mathrm{PNP}=3.74 \mathrm{MPa}$, leakage continues to decrease following the removal of ultrasound, resulting in a drop in fluorescence between ultrasound applications.

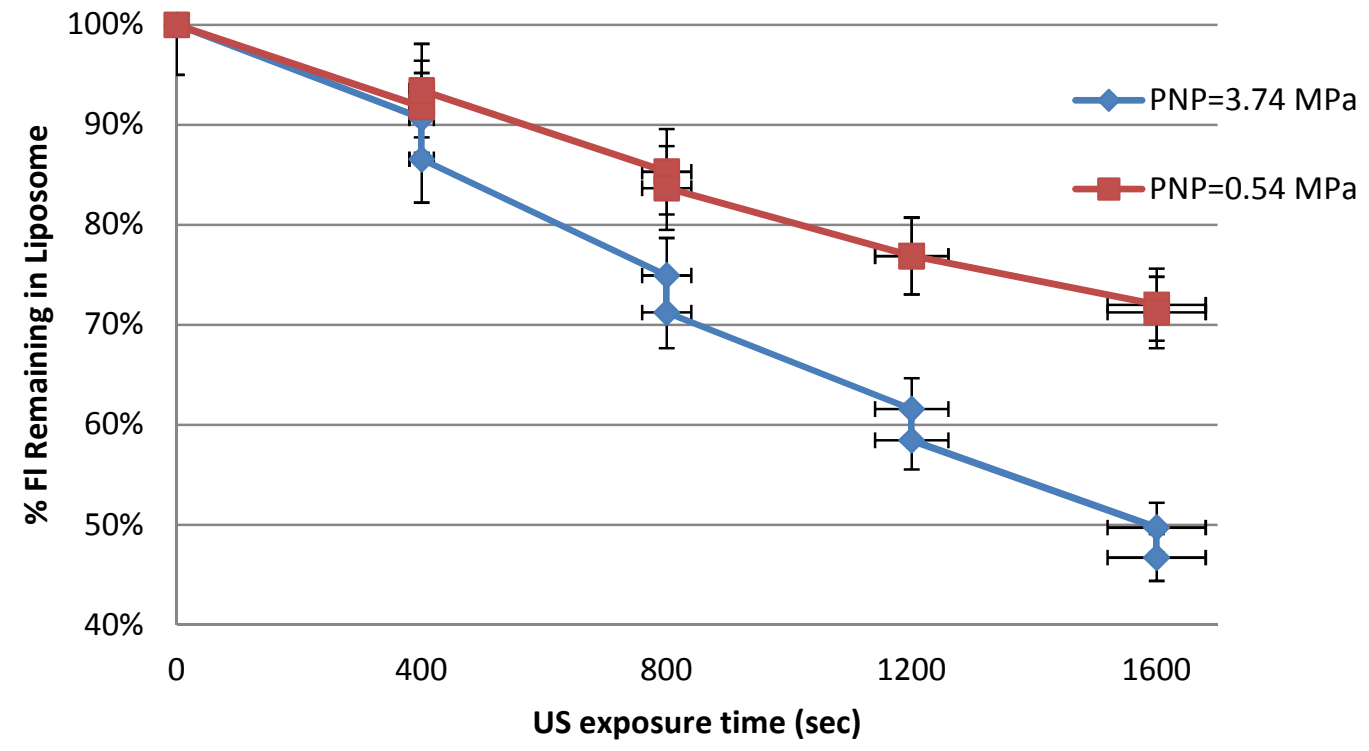

Figure 5: Ultrasound is applied with a $1 \mathrm{MHz}$ focused transducer. The sample is subjected to 400 sec of ultrasound exposure. At this point, ultrasound is turned off for $\mathbf{1 2 0}$ seconds to determine whether the percent of fluorescence remaining will continue to decrease or remain the same during this period which gives insight into the type of membrane disruption at each pressure. This process is repeated for a total of four periods of ultrasound application and subsequent removal. One sample is tested at low pressure $(0.54 \mathrm{MPa})$ where stable cavitation is occurring, while the other sample is done at high pressure (3.74 $\mathrm{MPa})$ where $35.62 \% \pm 6.32 \%$ of the microbubble population experiences inertial cavitation.

Changing ultrasound pressure amplitude is not the only factor in this system that has an effect on the type of cavitation experienced by the microbubble. Theoretical work involving a microbubble oscillating in the center of a rigid tube has led to predictions that a decrease in magnitude of microbubble oscillation and a change in resonance frequency will occur due to the restriction from a tube with a diameter of the same order of magnitude as the microbubble [3234]. Accordingly, it is reasonable to think that the interaction between two microbubbles would also suppress oscillations as shown experimentally with two UCA microbubbles trapped near a wall in the work by Garbin et al [35]. Therefore a smaller number of microbubbles within the liposome would lead to greater oscillations and the potential for increased membrane disruption. 
Our typical recipe for microbubbles nested within the liposomes requires a microbubble concentration of $5 \times 10^{8} \mathrm{MB} / \mathrm{mL}$. To determine if the amount of microbubbles used in the recipe influences the leakage behavior of the sample, the concentration of microbubbles used for each recipe was varied between $2 \times 10^{8}$ and $8 \times 10^{8} \mathrm{MB} / \mathrm{mL}$ and exposed to an ultrasound PNP of 1.54 $\mathrm{MPa}$. It is assumed that a greater concentration of microbubbles used in the recipe results in an increased number of microbubbles nested within the liposome, although the encapsulation efficiency remains unknown.

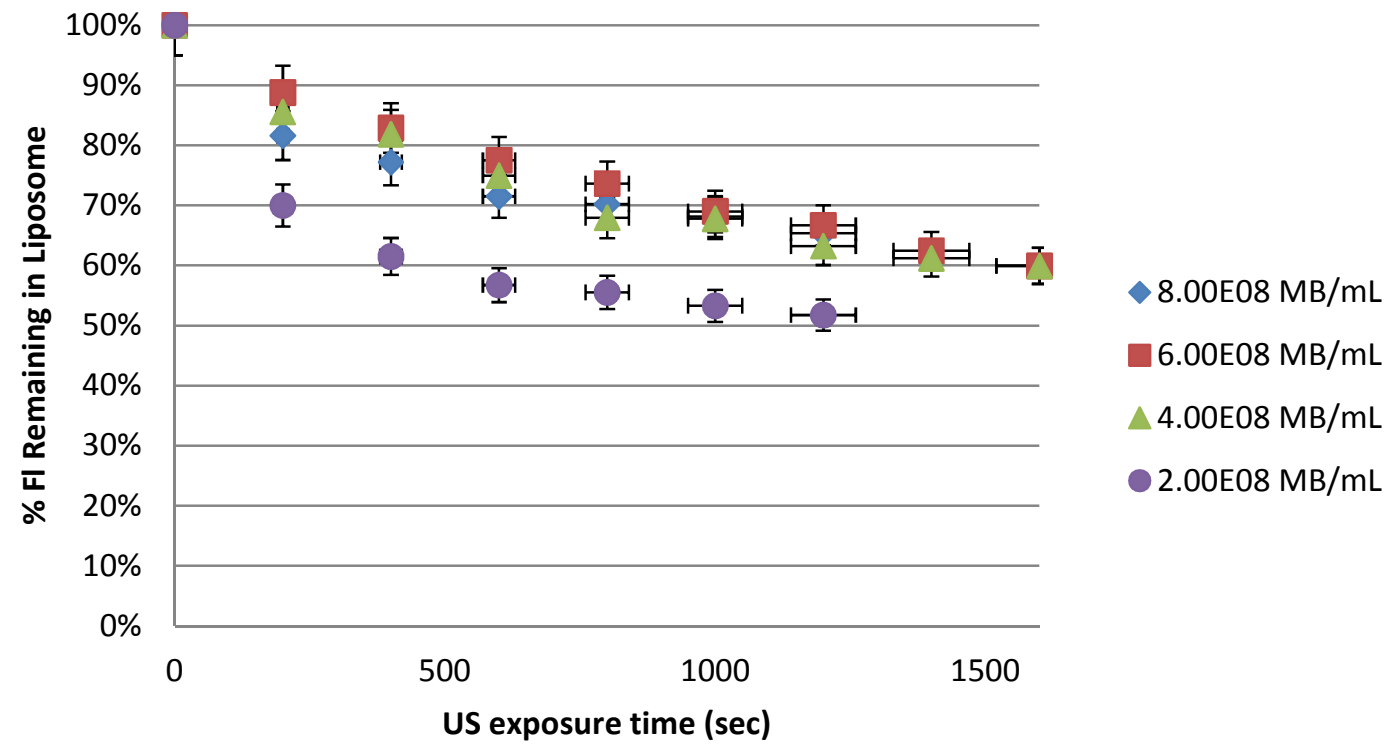

Figure 6: Samples are exposed to $1 \mathrm{MHz}$ ultrasound where the only variation between samples is the concentration of microbubbles used during preparation. The sample is exposed to an ultrasound PNP of 1.54 $\mathrm{MPa}$

Increasing the microbubble concentration from $4 \times 10^{8}$ to $8 \times 10^{8} \mathrm{MB} / \mathrm{mL}$ has no effect on leakage behavior, but below this at a concentration of $2 \times 10^{8} \mathrm{MB} / \mathrm{mL}$, a $13.66 \%$ decrease in fluorescence is observed at the conclusion of the experiment. This is consistent with increased activity found in other nested microbubble formulations for a decrease in microbubble concentration [25].

If microbubble repulsions affect the leakage profile as demonstrated in Figure 6, then we may also hypothesize that the repulsion between the liposome wall and microbubble wall will also affect the leakage profile. To investigate further, the average diameter of liposomes is varied between 4.74 and $6.04 \mu \mathrm{m}$ by changing the homogenizer speed while keeping the microbubble concentration constant at $5 \times 10^{8} \mathrm{MB} / \mathrm{mL}$ and exposing the sample to an ultrasound PNP of 1.54 MPa. 


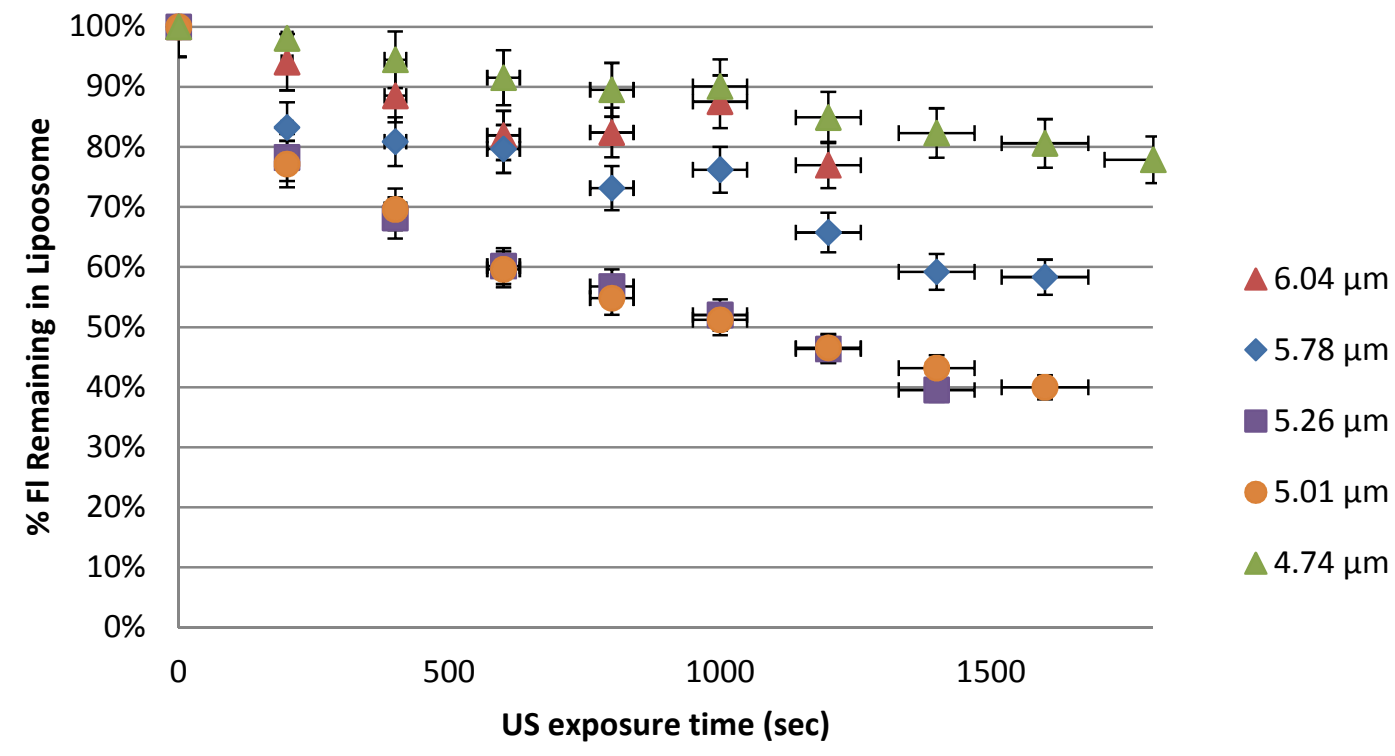

Figure 7: Samples are exposed to $1 \mathrm{MHz}$ ultrasound where the only variation between samples is the diameter of nesting shell used during preparation. The sample is exposed to an ultrasound PNP of 1.54 MPa

Upon inspection of Figure 7, we see that the liposomes with an average diameter of $5.01 \mu \mathrm{m}$ and $5.26 \mu \mathrm{m}$ give maximal leakage out of the size distributions examined which suggests that there may be an optimal ratio of microbubble diameter to liposome diameter.

\section{Discussion}

This study shows conclusively that focused ultrasound can be used to trigger release of the internal aqueous contents of a liposome. However, ultrasound alone is not sufficient to trigger release at clinically relevant (high frequency $\sim 1-10 \mathrm{MHz}$ ) conditions. Rather, content release is achieved via the presence of microbubbles nested inside of the liposome's aqueous core; ultrasound induces microbubble oscillations which in turn facilitate release. A question naturally arises, namely, what is the mechanism of release?

It is well documented that cavitating microbubbles cause membrane disruption as de Jong first disclosed with real-time, ultrafast transmission microscopy that the interaction between microbubbles and cell membrane was responsible for membrane poration [15, 16, 31, 36-41]. Microbubble oscillations are categorized as either stable or inertial cavitation and the type of cavitation determines the extent of membrane disruption. Non-inertial cavitation, or stable cavitation, is present when using low intensity ultrasound. Such stable oscillations are able to gently push and pull the membrane, inducing moderate and reversible changes at the cellular level, including opening cell walls and loosening tight junctions [36]. This increased permeability in the membrane allows a drug or drug mimic to enter the membrane, or in this case, be released from the interior of a liposome as long as the oscillating microbubbles are present [41]. This phenomenon is consistent with transient poration and/or dilation of the vesicle bilayer. 
If pressure amplitude is sufficiently high and above a certain threshold level, inertial cavitation is observed. When a collapsing microbubble is located close to a surface like a membrane, an asymmetrical collapse takes place, and results in the formation of a liquid jet towards the nearby surface [21]. It has been shown that these shock waves and microjets create very high forces which cause either permanent poration due to large lesions in the cell membrane or cell lysis in some cases due to the turbulence associated with bubble translation. Figure 8a illustrates dilation of the membrane while Figure $8 \mathrm{~b}$ illustrates poration, either temporary or permanent.

The drug mimic, calcein, used in this study is a fluorescent dye with a reported molecular radius of $0.65 \mathrm{~nm}$ [44]. If calcein is diffusing through a pore in the membrane, the pore need not be permanent. The diffusion length of calcein is $28.02 \mu \mathrm{m}$ for the negative amplitude of one ultrasound pulse which is orders of magnitude larger than the thickness of a lipid bilayer. Generally, pore sizes obtained with rather modest acoustic pressures were reported from several tens of nanometers to a few hundreds of nanometers [31]. The size of calcein presents no obvious complications for these studies, and diffusion through membrane pores can not be ruled out.

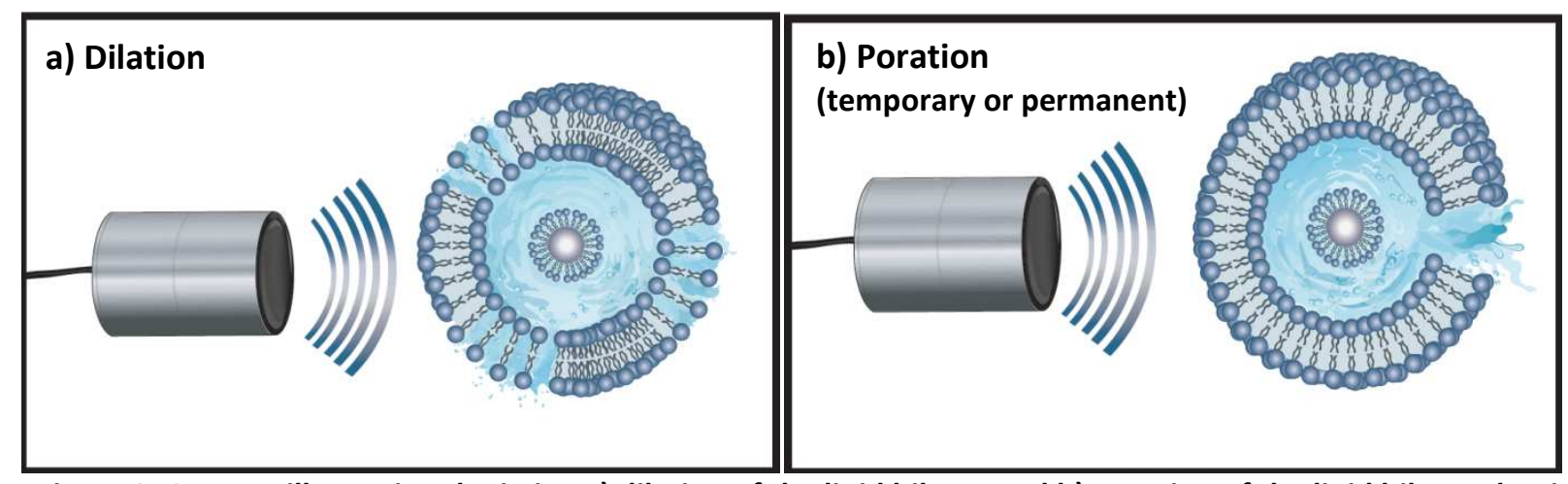

Figure 8: Cartoon illustration depicting a) dilation of the lipid bilayer and b) poration of the lipid bilayer that is either temporary lasting only while ultrasound is applied or permanent damage to the liposome bilayer believed to be caused by inertial cavitation of the microbubble. (Source: Jennifer Bing, Program Coordinator of Department of Chemical and Biological Engineering at Drexel University. 2014)

Figure 4 shows the results of a set of studies done for liposomes with an average diameter of $5.01 \mu \mathrm{m}$ where the percent of calcein remaining within the liposomes is observed for peak negative pressures between 0.54 and $3.74 \mathrm{MPa}$. The amount of release is not a linear function of ultrasound pressure; rather, the rate of calcein leakage seems to be controlled by the type of cavitation of the microbubble. It appears that two regimes exist with a threshold between 1.26 and 2.06 $\mathrm{MPa}$. Observations of thresholds associated with cavitation-induced biologic effects and other ultrasonic phenomena are not unusual and are very well documented [21, 31, 45-47]. For the purpose of this study, it has been confirmed that at the lowest peak negative pressure $(\mathrm{PNP}=0.54 \mathrm{MPa}) 0.52 \% \pm 5.69 \%$ of the sample is inertially cavitating and at the highest pressure $(\mathrm{PNP}=3.74 \mathrm{MPa}) 35.62 \% \pm 6.32 \%$ of the sample is inertially cavitating. A detailed analysis of this cavitation detection method that was used can be found in Mleczko, et al [27].

A one-way ANOVA test was done in conjunction with Tukey pairwise comparisons to determine with $95 \%$ confidence whether the percent of fluorescence remaining in the liposome after 200 
seconds of ultrasound exposure for peak negative pressures of 0.54 to $1.26 \mathrm{MPa}$ are statistically different. It was found that there is no statistical difference in this "low pressure" regime between samples at this time point, demonstrating that the leakage profile seems to be correlated with stable cavitation occurring in all low intensity experiments so long as the pressure amplitude is below the inertial cavitation threshold. There is also no statistical difference in the fluorescence measurements after 200 seconds from the "high pressure" regime with peak negative pressures between 2.06 and $3.74 \mathrm{MPa}$. This study seems to agree with two separate mechanisms differentiating between damage caused by stable cavitation versus inertial cavitation. The rate of decrease in fluorescence is slower $(-3.148 \%)$ at $0.54 \mathrm{MPa}$ for the first 1000 seconds of experimentation where less inertial cavitation events are detected and faster $(-4.877 \%)$ at 3.74 $\mathrm{MPa}$ where $35.62 \%$ of the microbubbles undergo inertial cavitation. Neither the amount of calcein released from the vesicle as well as ultrasound exposure time required to reach steady state do not seem to be correlated with ultrasound amplitude.

We deduce that leakage is caused by dilation, poration and/or catastrophic rupture of the membrane, where the extent of membrane disruption, either temporary or permanent, is dependent on acoustic pressure and therefore the type of cavitation- stable or inertial- that the microbubble undergoes. If leakage is due to dilation/poration caused by stable cavitation then we expect the temporal release profile to display a decrease in fluorescence remaining within the liposome during ultrasonic exposure then remain constant when ultrasound is removed for the duration of the experiment. If leakage is caused by permanent poration/rupture of the liposome due to inertial cavitation then we expect a decrease in fluorescence remaining within the liposome even after ultrasound is removed from the sample chamber.

To determine whether membrane disruption is permanent or temporary, ultrasound was turned on and off for intervals of $400 \mathrm{sec}$ of ultrasound exposure followed by $120 \mathrm{sec}$ of rest. Fluorescence was measured before after each interval of ultrasound application to determine if the transport of calcein from the interior of the liposome to the external solution would continue when ultrasound was removed. The study was done at $3.74 \mathrm{MPa}$ and $0.54 \mathrm{MPa}$ to determine if results were pressure dependent. Figure 5 proves that at low amplitude $(0.54 \mathrm{MPa})$, leakage only persists during ultrasound exposure. When fluorescent measurements were taken after ultrasound is removed, leakage ceased immediately proving that the pores forming and/or dilation of the membrane that allow for release of the liposome contents during ultrasound exposure are not permanent damage to the membrane. Fast resealing of cell membrane porations are reported to occur in the order of milliseconds to seconds [48-50] after switching off the ultrasound at relatively low pressures. The fact that cell membrane permeabilization is rapidly decaying indicates that pores exist as longs as the oscillating microbubbles are present [16, 38, 41, 51]. The pores forming in the membrane that allow for release of the liposome contents during ultrasound exposure are not permanent damage to the membrane.

However, Yudina et al. demonstrated that the cellular uptake of cell-impermeable small compounds persisted up to $24 \mathrm{~h}$ with a half-life of $8 \mathrm{~h}$ [15] at higher amplitudes so we also measured fluorescence of the sample for the highest peak negative pressure used in this study (3.74 MPa). Studies at high amplitude $(\mathrm{PNP}=3.74 \mathrm{MPa})$ show that leakage continues to occur during each period where ultrasound was turned off. This could be evidence that the disruption to the membrane is more extreme at these high pressures so a fraction of the sample is either 
destroyed or permanently porated due to inertial cavitation. The damaged vesicles continue to release their contents even during the fourth period of ultrasound removal (after $1600 \mathrm{sec}$ of ultrasound application). The data from the temporal release study agrees with the inertial cavitation study as evidenced by fluorescence decrease both during ultrasound application and while ultrasound is turned off. $35.62 \%$ of the sample is inertially cavitating, which likely owes to the decrease in fluorescence during periods of "rest" (result of permanent damage) while the remaining fraction of the sample experiences temporary membrane disruption and allows for release when ultrasound is reapplied.

Figures 6 and 7 were used to determine whether the microbubble concentration and liposome diameter affect leakage behavior. The identical profiles for both 5.01 and $5.26 \mu \mathrm{m}$ suggest that there may an ideal range of liposome size- if not an optimum- that allows for the most leakage when exposed to ultrasound. This is consistent with previous studies of nested microbubbles (although the microbubbles were nested within a polymer shell) that suggest a smaller concentration of microbubbles within the shell allows for greater microbubble expansion, leading to an increase in inertial cavitation events. This could explain an increase in leakage at lower microbubble concentrations. A one-way ANOVA combined with a Tukey pairwise comparison confirm that for concentrations $4 \times 10^{8}$ to $8 \times 10^{8} \mathrm{MB} / \mathrm{mL}$ there is no statistically significant difference in the percent fluorescence remaining after 1000 waveforms, but as seen on the graph, $2 \times 10^{8} \mathrm{MB} / \mathrm{mL}$ gives a different leakage profile. This concentration of microbubbles resulted in the most leakage which suggests that the microbubbles inside of the liposome cause sonoporation, not the ones existing outside of the liposome.

Microbubble concentration to liposome diameter ratio may also play a role when determining the desired leakage rate and final percentage of calcein remaining in the liposome, but further studies are needed to confirm this. While stably cavitating microbubbles need to be within 1 microbubble diameter from the cell to affect the membrane [47], the effects of inertially cavitating microbubbles reach over a larger distance. However, it was calculated that the maximal distance between microbubble and cell membrane should not exceed the microbubble diameter to have an effective impact on the cellular membrane [36]. We suspect there is a set of conditions that allow for maximum leakage and the highest leakage rate, which is dependent on the type of cavitation event (pressure dependent), liposome formulation, liposome diameter, and microbubble concentration. Microbubble size also presumably plays a role in the leakage behavior, but the microbubble samples used during experimentation had a Gaussian size distribution following formulation and were not further separated or filtered by size.

\section{Conclusion}

This study confirms that nested microbubbles are effective candidates for triggered drug delivery vehicles with an ultrasound trigger. The nested formulation has dual benefits: triggered release of contents of the aqueous core of the liposome and an increase in image brightness longevity. Triggered release can be accomplished with both high and low frequency transducers, although high frequency is of greater interest due to its potential use in a clinical setting owing to avoidance of deleterious effects to tissue. Low frequency experiments with an ultrasound probe operating at $20 \mathrm{kHz}$ proved that leakage was accomplished with and without microbubbles incorporated into the liposome core. 
An important aspect of this research is the relationship between the cavitation event and membrane disruption. Two regimes exist during the initial membrane disruption caused by cavitating microbubbles. A faster release rate, as well as permanent membrane damage is seen for samples exposed to high pressure (2.06 to $3.74 \mathrm{MPa}) 1 \mathrm{MHz}$ ultrasound transducer. A slower release rate and dilation/temporary poration is characteristic of stable cavitation for low pressure studies (0.54 to $1.74 \mathrm{MPa})$. Further research will include a thorough study of the type of cavitation (inertial versus stable) at each peak negative pressure. It is believed that at low pressures, stable cavitation within these liposomes occurs up to $1.26 \mathrm{MPa}$ which explains why the leakage behavior for samples at and below this pressure follow one profile, while leakage for pressures above this follow a different profile- one with a sharper initial decrease in fluorescence remaining within the liposome. It would be reasonable to assert that inertial cavitation is causing larger, more permanent membrane damage in accordance with research done by Lentacker et al. which results in a greater initial loss in fluorescence [31]. A detailed study evaluating the fraction of sample undergoing inertial cavitation for each peak negative pressure is imperative to understanding this novel drug delivery vehicle.

Future work may also include using different size molecules for the fluorescence quenching assay to determine pore size at each pressure amplitude. Generally speaking, pore sizes which have been reported as a consequence of inertial cavitation (hundreds of nanometers to micrometer range) are larger than pores reported during stable cavitation (few nm to hundreds of nanometers). Moreover, pore size has been shown to correlate with acoustic pressure; higher acoustic pressures result in larger microbubble oscillations and larger pores [52] and [53]. It is clear that the liposome shell, microbubble concentration, and ultrasound pressure amplitude all impact the leakage behavior of the nested microbubbles which gives insight into the inertial effect this has on the physics of the system. This study expands on the advantages of nested microbubbles as triggered drug delivery vehicles and provides insight as to their mechanism(s) of action.

\section{Acknowledgment}

This research was supported in part by grant 1064802 from the National Science Foundation. 


\section{References}

1. Bangham, A.D., M.M. Standish, and J.C. Watkins, Diffusion of univalent ions across the lamellae of swollen phospholipids. Journal of Molecular Biology, 1965. 13(1): p. 238-IN27.

2. Gregoriadis, G. and B.E. Ryman, Liposomes as carriers of enzymes or drugs: a new approach to the treatment of storage diseases. Biochem J, 1971. 124(5): p. 58P.

3. Immordino, M.L., F. Dosio, and L. Cattel, Stealth liposomes: review of the basic science, rationale, and clinical applications, existing and potential. Int J Nanomedicine, 2006. 1(3): p. 297-315.

4. Bibi, S., et al., Trigger release liposome systems: local and remote controlled delivery? J Microencapsul, 2012. 29(3): p. 262-76.

5. Price, R.J., et al., Delivery of colloidal particles and red blood cells to tissue through microvessel ruptures created by targeted microbubble destruction with ultrasound. Circulation, 1998. 98(13): p. 1264-7.

6. Yoon, Y.I., et al., Ultrasound-mediated gene and drug delivery using a microbubble-liposome particle system. Theranostics, 2014. 4(11): p. 1133-44.

7. Vignon, F., et al., Microbubble cavitation imaging. IEEE Trans Ultrason Ferroelectr Freq Control, 2013. 60(4): p. 661-70.

8. K. Ferrara, R.P., M. Borden, Ultrasound Microbubble Contrast Agents: Fundamentals and Application to Gene and Drug Delivery. Annual Review of Biomedical Engineering, 2007(9): p. 415-47.

9. Church, C.C. and E.L. Carstensen, "Stable" inertial cavitation. Ultrasound Med Biol, 2001. 27(10): p. $1435-1437$.

10. Leighton, T.G., The Acoustic Bubble1994, San Diego, CA: Academic Press.

11. Tung, Y.S., et al., Identifying the inertial cavitation threshold and skull effects in a vessel phantom using focused ultrasound and microbubbles. Ultrasound Med Biol, 2010. 36(5): p. 840-52.

12. Bao, S., B.D. Thrall, and D.L. Miller, Transfection of a reporter plasmid into cultured cells by sonoporation in vitro. Ultrasound Med Biol, 1997. 23(6): p. 953-9.

13. Greenleaf, W.J., et al., Artificial Cavitation Nuclei Significantly Enhance Acoustically Induced Cell Transfection. Ultrasound Med Biol, 1998. 24(4): p. 587-595.

14. Kim, H.J., et al., Ultrasound-mediated transfection of mammalian cells. Hum Gene Ther, 1996. 7(11): p. 1339-46.

15. Yudina, A., M. Lepetit-Coiffe, and C.T. Moonen, Evaluation of the temporal window for drug delivery following ultrasound-mediated membrane permeability enhancement. Mol Imaging Biol, 2011. 13(2): p. 239-49.

16. Zhou, Y., et al., The size of sonoporation pores on the cell membrane. Ultrasound Med Biol, 2009. 35(10): p. 1756-60.

17. Tachibana, K., et al., Induction of cell-membrane porosity by ultrasound. Lancet, 1999. 353(9162): p. 1409.

18. Mehier-Humbert, S., et al., Plasma membrane poration induced by ultrasound exposure: implication for drug delivery. J Control Release, 2005. 104(1): p. 213-22.

19. Ibsen, S., et al., A novel nested liposome drug delivery vehicle capable of ultrasound triggered release of its payload. J Control Release, 2011. 155(3): p. 358-66.

20. Kheirolomoom, A., et al., Acoustically-active microbubbles conjugated to liposomes: characterization of a proposed drug delivery vehicle. J Control Release, 2007. 118(3): p. 275-84.

21. Wrenn, S.P., et al., Bursting bubbles and bilayers. Theranostics, 2012. 2(12): p. 1140-59.

22. Dicker, S., et al., Coencapsulation of lipid microbubbles within polymer microcapsules for contrast applications. Bubble Science, Engineering \& Technology, 2011. 3(1): p. 12-19. 
23. Szoka, F., Jr. and D. Papahadjopoulos, Procedure for preparation of liposomes with large internal aqueous space and high capture by reverse-phase evaporation. Proc Natl Acad Sci U S A, 1978. 75(9): p. 4194-8.

24. Frokjaer, S., Double emulsion vesicles, in Liposomes: A practical approach, N.R.C. C., Editor 1990, Oxford University Press: New York. p. 68-70.

25. N. Wallace, S.D., S. P. Wrenn, Influence of nesting shell size on brightness longevity and resistance to ultrasound-induced dissolution during enhanced B-mode contrast imaging. Ultrasonics 2014(submitted for publication).

26. Wrenn, S., et al. Controlling cavitation for controlled release. in Ultrasonics Symposium (IUS), 2009 IEEE International. 2009.

27. Mleczko, M. and G. Schmitz. A method for the determination of the inertial cavitation threshold of ultrasound contrast agents. in Ultrasonics Symposium, 2008. IUS 2008. IEEE. 2008.

28. Dicker, S., Colloidal Science of Ultrasound Contrast Agents, in Chemical Engineering2012, Drexel University. p. 264.

29. Lentacker, I., et al., Design and Evaluation of Doxorubicin-containing Microbubbles for Ultrasound-triggered Doxorubicin Delivery: Cytotoxicity and Mechanisms Involved. Mol Ther, 2009. 18(1): p. 101-108.

30. Legay, M., et al., Enhancement of Heat Transfer by Ultrasound: Review and Recent Advances. International Journal of Chemical Engineering, 2011. 2011: p. 17.

31. Lentacker, I., et al., Understanding ultrasound induced sonoporation: Definitions and underlying mechanisms. Advanced Drug Delivery Reviews, 2014. 72(0): p. 49-64.

32. Prosperetti, A. and A. Lezzi, Bubble dynamics in a compressible liquid. Part 1. First-order theory. Journal of Fluid Mechanics, 1986. 168: p. 457-478.

33. Sassaroli, E. and K. Hynynen, Forced linear oscillations of microbubbles in blood capillaries. J Acoust Soc Am, 2004. 115(6): p. 3235-43.

34. Cui, J., et al., Bubble pulsations between parallel plates. J Acoust Soc Am, 2006. 119(4): p. 206772.

35. Garbin, V., Optical Tweezers for the Study of Microbubble Dynamis in Ultrasound, in Nanotechnology2006, Universit a degli Studi di Trieste: Trieste, Italy.

36. Zhou, Y., et al., Controlled permeation of cell membrane by single bubble acoustic cavitation. J Control Release, 2012. 157(1): p. 103-11.

37. Moosavi Nejad, S., et al., Optical observation of cell sonoporation with low intensity ultrasound. Biochemical and Biophysical Research Communications, 2011. 413(2): p. 218-223.

39. Prentice, P., et al., Membrane disruption by optically controlled microbubble cavitation. Nat Phys, 2005. 1(2): p. 107-110.

40. Huang, S.-L. and R.C. MacDonald, Acoustically active liposomes for drug encapsulation and ultrasound-triggered release. Biochimica et Biophysica Acta (BBA) - Biomembranes, 2004. 1665(1-2): p. 134-141.

41. van Wamel, A., et al., Vibrating microbubbles poking individual cells: Drug transfer into cells via sonoporation. Journal of Controlled Release, 2006. 112(2): p. 149-155.

42. VanBavel, E., Effects of shear stress on endothelial cells: Possible relevance for ultrasound applications. Progress in Biophysics \& Molecular Biology, 2007. 93(1-3): p. 374-383.

43. Wu, J., Theoretical study on shear stress generated by microstreaming surrounding contrast agents attached to living cells. Ultrasound in medicine \& biology, 2002. 28(1): p. 125-129.

44. Dijkink, R., et al., Controlled cavitation-cell interaction: trans-membrane transport and viability studies. Phys Med Biol, 2008. 53(2): p. 375-90. 
45. Dicker, S.M., M; Hensel, K; Bartolomeo, A; Schmitz, G; Wrenn, S P, Coencapsulation of lipid microbubbles within polymer microcapsules for contrast applications. Bubble Science, Engineering \& Technology, 2011. 3(1): p. 12-19.

46. Pong, M., et al., In vitro ultrasound-mediated leakage from phospholipid vesicles. Ultrasonics, 2006. 45(1-4): p. 133-145.

47. Tomita, Y., T. Matsuura, and T. Kodama, Temporal effect of inertial cavitation with and without microbubbles on surface deformation of agarose $\mathrm{S}$ gel in the presence of 1-MHz focused ultrasound. Ultrasonics, 2015. 55(0): p. 1-5.

48. Deng, C., et al., Ultrasound-induced cell membrane porosity. Ultrasound in medicine \& biology, 2004. 30(4): p. 519-526.

49. Zhou, Y., J. Cui, and C. Deng, Dynamics of sonoporation correlated with acoustic cavitation activities. Biophysical Journal, 2008. 94(7): p. 3.

52. Huber, P.E., et al., Focused ultrasound (HIFU) induces localized enhancement of reporter gene expression in rabbit carotid artery. Gene Ther, 2003. 10(18): p. 1600-7.

53. McCreery, T.P., R.H. Sweitzer, and E.C. Unger, DNA delivery to cells in culture using ultrasound. Methods Mol Biol, 2004. 245: p. 287-92. 